\title{
Tribotronic Planar Graphene Transistors for low-energy-consumption high/low frequent stimuli monitoring
}

\author{
Yanfang Meng ${ }^{1,2 *}$ \\ ${ }^{1}$ State Key Laboratory of Advanced Optical Communications System and Networks, School of Electronics Engineering and \\ Computer Science, Peking University, Beijing 100871, China. \\ ${ }^{2}$ Center for Flexible Electronics Technology, Tsinghua University, Beijing, 100084, China
}

\begin{abstract}
Asian Journal of Complementary and Alternative Medicine. Volume 10 Issue 01
\end{abstract}
Published on: 12/01/2022

*Author for Correspondence: Yanfang Meng, Center for Flexible Electronics Technology, Tsinghua University, Beijing, 100084, China; Tel: 1880329732; Email: yanaimengmeng@126.com

Cite this article as: Meng Y. Tribotronic Planar Graphene Transistors for low-energy-consumption high/low frequent stimuli monitoring. Asian Journal of Complementary and Alternative Medicine, Vol 10(1), 2-4:2021.

The developments of flexible electronic skins (e-skins), epidermal electronics and mechanosensation active matrix with the aim of miming perceptive functions of human skin, have inspired great research interests for potential applications in robotics, prostheses, and wearable healthcare monitoring devices [1]. The above device were in function through converting the external stimuli into quantified physical signals and further provide feedback instructions. [2-7] Mechanosensation matrix mainly concentrated on replication of human somatosensory system to sense various mechanical stimuli.[8-11] Scientists and researchers dedicated their great efforts on materials design and structures optimization [12-10] towards mechanosensation active matrix to realized high sensitivity, fast response time, mechanical flexibility, and durability multimodal sensing for practical noninvasive applications for long term durability, multimodal sensing and highly integrated, simplify fabrication process, and minimizing the power consumption [15-18].

Recently, based on their previous work [19],writing in Scientific Reports, Yanfang Menga, b* and Guoyun Gao have been proposed a self-powered bifunctional sensor based on tribotronic planar graphene transistors, which was employed to real-time monitoring with high energy-efficient and $2 \mathrm{D}$ imaging [20].

To achieve low energy-consumption of the device, tribopotential-powered GFET is presented with the mechanism involved triboelectric and electrostatic inducing. Apart from energy-efficiency, their work also realized the perception of multiple stimuli (high frequent stimuli and low frequent stimuli) to emulate the human's skin, which is also of great significance issue on schedule [21].

In regard to the low frequent stimuli, magnetic field sensor as a model, whose work principal was that (magnetic film and ion gel as friction layer) the magnetic field changed the distance between magnetic film and ion gel, giving rise to different signal. (Our previous work verified that the output of tribopotential-powered GFET had certain functional relationship with the triboelectric distance).

Given that skin can percept both high frequent and lower frequent stimuli [22], and it was difficult to detect magnitude of frequency of the high frequent stimuli intuitively. To solve this challenge, we adopted the methodology of plasticity behaviour synaptic transistor (transistor served both synaptic transistor and sensing unit). In synaptic transistor, synaptic weight (the increment phenomenon of later post-synaptic current compared with former counterpart) varied with the variation of frequency of pre-synaptic stimuli (signal) [23]. In this work, wind was recognized as a high-frequency stimuli source (the wind stimuli was employed wind driving friction of electrofrictional layer of TENG), the increment of postsynaptic current of No. $n$ pulse compared with No. $n-1$ pulse was utilized to indicate the real-time frequency of wind that the sensor experienced.

Incorporating synaptic transistor into E-skin has already been studied. Ravinder reported a printed synaptic transistor to create E-skin with positive mind and opened up a novel reformation: inserting Neuro layer into E-skin to advance intelligentialization and can immediately respond to external stimuli in absence of instruction. This project would spur the reformation of wearable technology, flexible electronic and new generation computer [23]. Furthermore, graphene was selected as channel semiconductor for high mobility (chemical vapour deposited (CVD) graphene with mobility approaching $200000 \mathrm{~cm}^{2} \mathrm{~V}^{-1} \mathrm{~s}^{-1}$ for the carrier density below 5 $\times 109 \mathrm{~cm}^{-2}$ at a low temperature [25]), high transparency (the white light absorbance of a suspended graphene monolayer is 
2.3\% (or transmittance of 97.7\%) with a negligible reflectance of $<0.1 \% 26$ ), high Young's modulus ( 1 TPa) [27] excellent thermal stability and conductivity $\left(3000 \sim 5000 \mathrm{~W} \mathrm{~m}^{-1} \mathrm{~K}^{-1}\right)$ [28], and peculiar surface area[29].Therefore, graphene field effect transistor (GFET) applied as synaptic has reports [30].

With the development material science, electronics and energy science, the self-powered sensors based on tribotronic with mutilfunctional will be given considerable attention to fulfill the ever-growing demands of human-machine interactive interface, and point of care of surveillance and artificial electronic skin. The great progresses have been made to improve the properties of self-powered sensor based on tribotronic. Our work would offer a plausible platform for intelligentization of E-skin.

However, there are still many challenges for the development of self-powered sensors based on tribotronic. First, the interpretation of principle of relationships between the microstructure and properties is deficiency. Second, the optimum points of synergistic interactions among the multiple factors are supposed to be unambiguity. Third, the meet the new demands of area of sophisticated equipment, the comprehensive properties combining ultrahigh sensitivity, low energy consumption, low damping, outstanding mechanical and flexibility are supposed to be explored urgently.

\section{REFERENCES}

1. Park J, Lee Y, Ha M, Cho S, Ko H(2016). Micro/Nanostructured Surfaces for Self-Powered and Multifunctional Electronic Skins. J. Mater. Chem. B, 4: 2999-3018.

2. Someya T, Sekitani T, Iba S, Kato Y, Kawaguchi H, Sakurai T.(2004) A large-area, flexible pressure sensor matrix with organic field-effect transistors for artificial skin applications. Proceedings of the National Academy of Sciences of the United States of America, 101:9966-9970.

3. Sekitani $\mathrm{T}$, Someya $\mathrm{T}(2012)$ Stretchable organic integrated circuits for large-area electronic skin surfaces. MRS Bull. 37:236-245.

4. Schwartz G, Tee B C K, Mei J, Appleton A L, Kim D H, Wang H, Bao $Z(2013)$ Flexible polymer transistors with high pressure sensitivity for application in electronic skin and health monitoring. Nat. Commun, 1859.

5. Wang X, Gu Y, Xiong Z, Cui Z, Zhang T(2014) Silk-Molded Flexible, Ultrasensitive, and Highly Stable Electronic Skin for Monitoring Human Physiological Signals. Adv Mater, 26:1336-1342.

6. Bauer S(2013) Flexible electronics: Sophisticated skin, Nat Mater, 12:871-872.

7. Ramuz M, Tee B.C.K., Tok J.B.H., Bao Z(2012) Transparent, Optical, Pressure-Sensitive Artificial Skin for Large-Area Stretchable Electronics. Adv. Mater. 24:3223-3227.

8. Sun Q, Seung W, Kim B J, Seo S, Kim S W, Cho J H(2015) Active Matrix Electronic Skin Strain Sensor Based on Piezopotential-Powered Graphene Transistors. Adv. Mater. 27:3411.

9. Zhang Q, Jiang T, Ho D, Qin S, Yang X, Cho J H, Sun Q, Wang Z L(2018) Transparent and Self-Powered Multistage Sensation Matrix for Mechanosensation Application. ACS nano, 12:254.
10. Sun Q, Ho D H, Choi Y, Pan C, Kim D H, Wang Z L,Cho J H(2016) Piezopotential-Programmed Multilevel Nonvolatile Memory As Triggered by Mechanical Stimuli. ACS Nano 10:11037.

11. Zhao J, Li N, Yu H, Wei Z, Liao M, Chen P, Wang S, Shi D, Sun Q, Zhang G(2017) Static and Dynamic Piezopotential Modulation in PiezoElectret Gated MoS2 Field-E? ect Transistor. Adv. Mater. 29:1702076.

12. Yeo WH, Kim Y S, Lee J, Ameen A, Shi L, Li M, Wang S,Ma R, Jin S H, Kang Z, Huang Y, Rogers J A(2013) Multifunctional epidermal electronics printed directly onto the skin. Adv. Mater. 25: 2773.

13. Kim J, Lee M, Shim H J, Ghaffari R, Cho H R, Son D, Jung Y H, Soh M, Choi C, Jung S, Chu K, Jeon D, Lee S T, Kim J H, Choi S H, Hyeon T, Kim D H(2014) Stretchable Heater Using Ligand-Exchanged Silver Nanowire Nanocomposite for Wearable Articular Thermotherapy. Nat. Commun. 5:5747.

14. Ramuz M, B. C. K. Tee, J. B. H. Tok, Bao Z(2012) Transparent, Optical, Pressure-Sensitive Artificial Skin for Large-Area Stretchable ElectronicsAdv. Mater. 2012, 24, 3223.

15. Wang X, Gu Y, Xiong Z, Cui Z, Zhang T(2014). Silk-Molded Flexible, Ultrasensitive, and Highly Stable Electronic Skin for Monitoring Human Physiological Signals. Adv. Mater. 26: 1336-1342.

16. Pan L, Chortos A, Yu G, Wang Y, Isaacson S, Allen R, Shi Y, Dauskardt R, Bao Z(2014) An Ultra-Sensitive Resistive Pressure Sensor Based on Hollow-Sphere Microstructure Induced Elasticity in Conducting Polymer Film. Nat. Commun. 5: 3002.

17. Schwartz G, Tee B. C. K, Mei J, Appleton A L, Kim D H, Wang H, Bao Z(2013) Flexible Polymer Transistors with High Pressure Sensitivity for Application in Electronic Skin and Health Monitoring. Nat. Commun. 4:1859.

18. Mannsfeld S C, Tee B C, Stoltenberg R M, Chen C V H, Barman S, Muir B V, Sokolov A N, Reese C, Bao Z(2010) Highly Sensitive Flexible Pressure Sensors with Microstructured Rubber Dielectric Layers. Nat. Mater. 9:859-864.

19. Meng Y F, Zhao J Q, Yang X X, Zhao C L, Qin S S, Cho J H, Zhang C, Sun Q J, Wang Z L (2018) Mechanosensation-Active Matrix Based on DirectContact Tribotronic Planar Graphene Transistor Array. ACS Nano 12: 9381-9389.

20. Meng Y F, Gao G Y, Zhu J X(2021) Self-powered bifunctional sensor based on tribotronic planar graphene transistors. Scientific Reports 11: 21483.

21. Ho D H, Sun Q J, Kim SY, Han J T, Kim D H, Cho J H(2016) Stretchable and Multimodal All Graphene Electronic SkinAdv. Mater. 28:2601-2608.

22. Chun K Y, Son Y J, Jeon E S, Lee S, Han C S(2018) A Self-Powered Sensor Mimicking Slow- and Fast-Adapting Cutaneous Mechanoreceptors. Adv. Mater. 30: 1706299

23. Wan C J, Zhu L Q, Liu Y H, Feng P, Liu Z P, Cao H L, Xiao P, Shi Y, Wan Q(2016) Proton-Conducting Graphene Oxide-Coupled NeuronTransistors for Brain-Inspired Cognitive Systems. Adv. Mater. 28: 3557-3563.

24. Yelalwar R G, Ravinder Y(2018) Artificial Neural Network Based Approach for Spectrum Sensing in Cognitive Radio. International Conference on Wireless Communications, Signal Processing and Networking (WiSPNET):5.

25. Du X, Skachko I, Barker A, Andrei E Y(2018) Approaching ballistic transport in suspended graphene. Nat. Nanotechnol. 3:491.

26. Nair R R, Blake P, Grigorenko A N, Novoselov K S, Booth T J, Stauber T, Peres N M R, Geim A K(2008) Fine Structure Constant Defines Visual Transparency of Graphene. Science 320:1308. 
27. Lee C, Wei X D, Kysar J W, Hone J(2008) Measurement of the elastic properties and intrinsic strength of monolayer graphene. Science 321:385.

28. Balandin A A, Ghosh S, Bao W, Calizo I, Teweldebrhan D, Miao F, Lau C $\mathrm{N}(2008)$ Superior thermal conductivity of single-layer graphene. Nano Lett. 8: 902.
29. Stoller M D, Park S, Zhu Y, An J, Ruoff R S(2008) Graphene-based ultracapacitors. Nano Lett. 8:3498.

30. Sharbati M T, Du Y H, Torres J, Nolan D, Yun A M, Xiong F(2018) LowPower, Electrochemically Tunable Graphene Synapses for Neuromorphic Computing. Adv. Mater. 1802353. 